\title{
Perceptive Media - Adaptive Storytelling for Digital Broadcast
}

\author{
Adrian Gradinar ${ }^{1}$, Daniel Burnett ${ }^{1}$, Paul Coulton ${ }^{1}$, Ian Forrester ${ }^{2}$, Matt Watkins ${ }^{3}$, Tom \\ Scutt $^{3}$, Emma Murphy ${ }^{4}$ \\ ${ }^{1}$ Lancaster Institute for the Contemporary Arts, Lancaster University, Lancaster, UK \\ \{a.gradinar, d.burnett, p.coulton\}@lancaster.ac.uk \\ ${ }^{2}$ BBC R\&D North lab, Mediacity, UK \\ ian. forrester 1 @bbc.co.uk \\ ${ }^{3}$ Mudlark, Sheffield, UK \\ \{matt.watkins, tom.scutt\} @wearemudlark.com \\ ${ }^{4}$ Institute of Design Innovation, School of Design, Glasgow School of Art, Glasgow, UK \\ e.murphy@gsa.ac.uk
}

\begin{abstract}
Storytelling techniques within traditional broadcast media have not made major advances in recent years due to the linear and relatively rigid approach to narrative despite advances in the technology that delivers the content. This research proposes the concept of 'perceptive media' in which the content creators have at their disposal different tools and sensors to allow for the subtle adaption of the narrative without any direct interactions from the audience members. The concept is demonstrated through the creation of a 'perceptive radio' that is able to play specially designed content that adapts to the physical and social context in which the radio resides.
\end{abstract}

Keywords: Adaptive narrative, digital storytelling, context-aware media, perceptive media, perceptive radio.

\section{Introduction}

The storyteller greets the group slowly easing them into a relaxed ambiance where everyone is preparing to enjoy a well-told tale. As the story progresses, the storyteller adapts it to the particularities of the location and the group, making use of specific expressions, sayings or habits, referencing landmarks and places in the local vicinity to ensure the story resonates with the audience. The narrator also provides a warm welcome as more listeners join in, as well as becoming more engaged with the audience if their attention is drawn away from the story. It is through the use of these refined techniques, a personalized, unobtrusive storytelling practice is achieved, one where the subtle changes used by the storyteller contribute to the overall immersion level of the audience. In a similar vein, a well-versed stand-up comedian adapts their show to the specifics of the location of the theatre where the performance is taking place, tailoring the jokes around the audience and own perception of how well the 
audience is engaging with the show. Whilst the show is generally regarded as a performance, with an entertaining lead actor, the audience is experiencing a narrative given by a professional storyteller.

Despite the rapid pace of technological development across different mediums, broadcast storytelling content is still inflexible in comparison to these oral traditions. One medium that can adapt is digital games where game developers often create quite complex and twisting stories in which the player's actions, across diverse situations, directly influence the progression of the story as well as the evolution of the character and thus deliver a unique experience to each individual player (Mass Effect franchise $^{1}$, TallTale: The Walking Dead ${ }^{2}$, Alpha Protocol ${ }^{3}$ ). Whilst this technique has also been adapted to theatre by companies such as Punchdrunk ${ }^{4}$, it has yet to be seen in traditional broadcast media.

The main advances we have witnessed recently in broadcast media have only affected the way that we consume the digital content (TV, stream box, mobile, laptop) or how it is being delivered (on-demand media versus traditional media). Whilst online voting can change the outcome of a TV episode or show, no real advances have been made around how program makers are looking at making their content more dynamic and adaptable. Therefore, this research explores different ways in which content creators can start thinking about how they may produce adaptable stories, by making subtle changes to the storyline based on the context in which the listeners are consuming the media. In doing so, the content creators are generating a new form of media that within this research we referred to as 'perceptive media'.

Since there is little direct experience of creating such media, a research through design methodology [1] has been used to facilitate an exploratory process in which different technologies are used independently and concurrently to gather contextual information about the current context of the audience in order to influence specially created media. To this end various physical and digital sensors have been encapsulated into a physical device that is able to deliver this dynamic radio content and which we refer to as 'perceptive radio'.

\section{Perceptive Media}

Unlike interactive narrative, "in which users create or influence a dramatic storyline through their actions" [2], 'perceptive media' looks at narrative from a different perspective; it adapts a story to the audience by using information about them, gathered through the use of a range of sensors and sensing technologies, whilst shaping the story within the predefined scope set by the storyteller. If the goal of the interactive narrative is "to immerse users in a virtual world such that they believe that they are an integral part of an unfolding story and that their actions can significantly alter the direction or outcome of the story" [2], in the case of the 'perceptive media', the

\footnotetext{
http://masseffect.bioware.com/

https://www.telltalegames.com/

http://uk.ign.com/games/alpha-protocol/

4 http://punchdrunk.com/
} 
overall story arc does not change, it is simply varying the more ambient parts of the narrative to create an engaging experience for the listeners. Thus these changes may not be immediately apparent to the user and rather they are subtle and awareness may develop slowly during the course of the broadcast. Therefore, 'perceptive media' attempts to bridge the gap between the 'flexibility' of the storyteller and the 'rigidness' of the broadcasted story delivered. The technologies employed take advantage of the implicit and explicit data the audience is generating and, in doing so, it redefines narrative as a set of logical attributes (variables), which could be moulded into different experiences. It is through the creation of adaptable stories and new experiences that the concept of 'perceptive media' is achieved.

Since the story has to adapt to the contextual information gathered by the available sensors, new requirements are being placed on content creators. Whilst they have the unique opportunity to create new experiences, they are also required to generate the essential materials to cover all possible scenarios for a particular story. Each sensory output needs to be thoroughly considered before being used within a broadcast and it is within the responsibility of the content creators to ensure the correct usage of the sensors.

\section{The Perceptive Radio}

Currently, the 'perceptive media' concept takes the shape of a radio, that expands on the capabilities and content of the original Perceptive Radio developed by the BBC R\&D Department at MediCityUK in $2013^{5}$. Presently the range of sensors available consists of a microphone, used for noise cancellation, a camera, used to detect the number of people facing the radio, and a mobile phone, which is used to locate the radio. Based on the currently obtained information, extrapolated data is easily acquired, such as the weather, 'felt' temperature, 'real-feel' temperature or the time of day. Every piece of information could easily contribute to an even deeper personalisation level.

All the contextual information gathered through the sensors is passed to the radio, which employs a node.js server running on top of an Ubuntu distribution and delivering independent access to the sensors through an application program interface (API) built in JavaScript. The current setup was chosen to allow for the development of an easily expandable system, where other sensors could be effortlessly integrated. Furthermore, as the API is built using one of the most commonly used programming languages ${ }^{6}$ it permits for the rapid prototyping of new ideas content creators could design.

The final aim of the 'perceptive radio' is to demonstrate that the linear storytelling techniques developed within traditional broadcast media can be raised to a higher level, where contextual information can have a major contribution to the overall immersion level of the audience. The generated materials and concepts of this research

\footnotetext{
5 http://cubicgarden.com/2013/05/22/perceptive-radio/

6 http://www.tiobe.com/index.php/content/paperinfo/tpci/index.html
} 
will be made publicly available to encourage content creators to think of new ways in which digital stories are produced and delivered to the audience.

\section{A Campfire Story}

To demonstrate the notion of 'perceptive media' to both audience and content producers, the current prototype uses a storytelling narrative in the form of a 'campfire story', in particular an adaptation of one of the most well known Aesop's fables: The Two Travellers and the Farmer. The first contextual information gathered by the radio is its own location, by using the GPS position provided by the mobile phone. Based on this location, data such as the current weather and ' $f e l t$ ' temperature are obtained from online weather services. The time of day is also acquired from the internal clock alongside with the number of people currently within the sight of the camera. Based on this data, the story is dynamically generated. The 'campfire story' starts by greeting the listeners acknowledging their numbers, the time of day and ' $f e l t$ ' temperature. During the play, the radio monitors the activity of the listeners and the 'virtual storyteller' greets the listeners as they walk in or out of the room.

Similar to the original Aesop's fable, the 'virtual storyteller' asks each traveller about where they came from. Since the radio already has the contextual information about the location of the broadcast, the replies given by the travellers in the story reflect the current location. This is a subtle adaptation of the story, one that might not be perceived by all members of the audience; but, if registered by some of the listeners, it could enhance the overall experience.

\section{Conclusion}

The current iteration of this research introduces the concept of 'perceptive media', a new type of broadcast media, which makes use of contextual information provided through a range of different physical and digital sensors, to create a more engaging experience for the listeners by simply varying some of the ambient parts of the narrative. To demonstrate this concept a 'perceptive radio' has been developed. Through the use of a revised fable, the radio delivers an adapted narrative based on the location of the play, number of people in the room, time of day and weather data collected through the available sensors. In future work, the 'perceptive radio' will go under scrutiny from both content creators as well as the audience listening to the plays and conclusions will be drawn on the impact the 'perceptive media' makes on both categories involved.

\section{References}

1. Gaver, W.: What should we expect from research through design?. CHI '12. ACM, New York, NY, USA, 937-946, (2012).

2. Riedl, M.O., Bulitko, V.: Interactive Narrative: An Intelligent Systems Approach, AI Magazine, Vol. 34, No. 1, (2013). 